\title{
Delay-Constrained Beamforming and Resource Allocation in Full Duplex Systems
}

\author{
Mahmoud T. Kabir, Student Member, IEEE, and Christos Masouros, Senior Member, IEEE
}

\begin{abstract}
This paper studies the beamforming and resource allocation problem in a multiuser full duplex (FD) system with delay-awareness. We design a power-efficient algorithm to minimize the long-term sum transmit power under delay constraints. We do this by jointly optimizing the uplink transmit power and the downlink beamforming vectors while satisfying the long-term stability constraints on the queue buffers for the downlink and uplink users as well as quality of service constraints. Due to the stochastic nature of the problem, we exploit the classic drift-pluspenalty function and subsequently simplify the problem into a difference of convex functions. Building upon the transformed problem, we propose two algorithm designs, one, that exploits the users with good channel conditions for efficient resource allocation, and the other that ensures delay-fairness among all users based on the max-min formulation. Simulation results show significant gains achieved by the proposed FD schemes compared with the baseline HD schemes.
\end{abstract}

Index Terms-full-duplex, queue, stability, power minimization

\section{INTRODUCTION}

The next generation wireless networks are expected to provide high data rates and reliability for data transmission and various applications. Full duplex (FD) is one enabler of higher data rate communication. Thanks to the development of various self-interference (SI) cancellation/suppression techniques [1], FD is now closer to reality than ever. Motivated by the fact that the rapid growth of high data rate services leads to the increase in the power consumption in communication networks, it has become important to design power efficient communication systems in order to reduce the amount of greenhouse gases emission and operational expenses of communication systems.

The main aim in traditional system design is to minimize the system power consumption while satisfying some quality of service (QoS) constraints [2]. For example, [3] studied the resource allocation for distributed antenna systems with a FD base station (BS) that simultaneously serves uplink and downlink users where the network power consumption is minimized by jointly optimizing the downlink beamformer and uplink transmit power. Similarly, in [4], the authors investigated a power efficient resource allocation design for secure communications in a similar FD system setup. Also, the trade-off between the uplink and downlink power consumption was investigated in [4], [5]. In [5], the authors used the knowledge of the downlink signals at the FD BS to exploit multi-user interference (MUI) instead of treating the MUI as unwanted as in traditional interference suppression techniques.

However, with the rise of ultra reliable low latency communications (URLLC), a key performance metric with rising

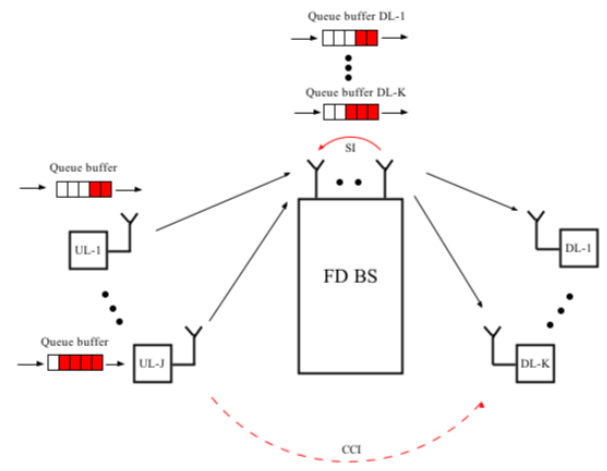

Fig. 1: A FD BS serving multiple users showing their queue buffers

interest towards $5 \mathrm{G}$ communications that has been neglected in traditional system designs is the transmission delay. Delay is a critical performance metric that determines the reliability of a wireless system and it is directly related to the system queue length [6]. [7] studied the trade-off between energy efficiency (EE) and delay in time-varying wireless systems by maximizes the system EE subject to network stability constraints. Similarly, in [8] the authors studied EE maximization in a buffferaided relay system while maintaining queue stability. In [9], the authors studied resource allocation in LTE-A system by maximizing a utility function of the transmission rate with queue stability constraints.

In contrast to the above mentioned works, which study either resource allocation in FD system without stability constraints or half-duplex (HD) resource allocation with stability constraints, we design a power-efficient resource allocation algorithm in multi-user FD system. The contributions of our proposed work as compared to existing works are twofold: (i) Unlike existing FD works in [3]-[5], we aim at minimizing the long-term average system transmit power while ensuring system queue stability, and, (ii) we jointly optimize the downlink beamforming vectors and uplink transmit power since existing HD methods in [7]-[9] can not be applied to our considered problem due to the introduction of SI and cochannel interference (CCI) in our FD study, which is not trivial to handle given the nature of the problem. Thus, in this paper we aim at addressing these gaps by means of weighted and delay constrained optimization.

\section{System Model}

We consider a multiuser communication system where a FD BS with $N$ transmit and $N$ receive antennas simultaneously serves $K$ single-antenna downlink users and $J$ uplink users 
[3]-[5]. In the considered system, we assume communication is time slotted and slot $t$ refers to the interval $[t, t+1)$, where $t \in$ $\{0,1,2, \ldots\}$. Accordingly, by letting $\mathbf{h}_{k} \in \mathbb{C}^{N \times 1}$ and $\mathbf{g}_{j} \in \mathbb{C}^{N \times 1}$ be the channels between the FD BS and the $k$-th downlink user, the $j$-th uplink user, respectively, the downlink and uplink transmission rates at slot $t$ in unit of bits/slot/ $\mathrm{Hz}$ are defined respectively by

$$
\begin{aligned}
& R_{\mathrm{DL}, k}(t)=\log _{2}\left(1+\gamma_{\mathrm{DL}, k}\right), \\
& R_{\mathrm{UL}, j}(t)=\log _{2}\left(1+\gamma_{\mathrm{UL}, j}\right),
\end{aligned}
$$

where

$$
\begin{aligned}
\gamma_{\mathrm{DL}, k}(t) & =\frac{\left|\mathbf{h}_{k}^{H} \mathbf{w}_{k}\right|^{2}}{\sum_{i \in\{1, \ldots, K\} / k}^{K}\left|\mathbf{h}_{k}^{H} \mathbf{w}_{i}\right|^{2}+\sum_{j=1}^{J} P_{\mathrm{UL}, j}\left|\ell_{j, k}\right|^{2}+\sigma_{k}^{2}}, \\
& =\frac{\mathbf{h}_{k}^{H} \mathbf{W}_{k} \mathbf{h}_{k}}{\sum_{i \in\{1, \ldots, K\} / k}^{K} \mathbf{h}_{k}^{H} \mathbf{W}_{i} \mathbf{h}_{k}+\sum_{j=1}^{J} P_{\mathrm{UL}, j}\left|\ell_{j, k}\right|^{2}+\sigma_{k}^{2}}, \\
\gamma_{\mathrm{UL}, j}(t) & =\frac{P_{\mathrm{UL}, j}\left|\mathbf{g}_{j}^{H} \mathbf{u}_{j}\right|^{2}}{\sum_{n \in\{1, \ldots, J\} / j}^{J} p_{n}\left|\mathbf{g}_{n}^{H} \mathbf{u}_{j}\right|^{2}+s_{j}+\sigma_{j}^{2}\left\|\mathbf{u}_{j}\right\|^{2}} .
\end{aligned}
$$

are the signal-to-interference plus noise ratio (SINR) [4], [5] at the $k$-th downlink user and at the FD BS, respectively. In (3) and (4), $\mathbf{w}_{k} \in \mathbb{C}^{N \times 1}, P_{\mathrm{UL}, j}$ and $\ell_{j, k}$ denote the beamforming vector for the $k$-th downlink user with $\mathbf{W}_{k}=\mathbf{w}_{k} \mathbf{w}_{k}^{H}$, the $j$-th uplink transmit power and the channel between the $j$ th uplink user and the $k$-th downlink user, respectively. In addition, $n_{k} \sim \mathcal{C N}\left(0, \sigma_{k}^{2}\right)$ and $\mathbf{n}_{j} \sim \mathcal{C N}\left(0, \sigma_{j}^{2}\right)$ are the additive white Gaussian noise at the $k$-th downlink user and the FD BS, respectively, and $\mathbf{u}_{j} \in^{N \times 1}$ is the receive beamforming vector for the $j$-th uplink user. In this paper, to reduce complexity, we adopt zero-forcing ( $\mathrm{ZF}$ ) receive beamforming at the FD BS for the detection of the uplink signals. ZF is adopted since it provides a good trade-off between complexity and performance [10].

In this paper, we assume the FD BS has perfect knowledge of channel state information (CSI) for the uplink and downlink users. We focus on slow fading channel scenario, where the channels change at the beginning of each frame. Thus, to facilitate the channel realization in practice, handshaking is performed between the FD BS and the uplink and downlink users. As pilot signals are usually embedded in the data packets and sent to the FD BS by the uplink users, and the downlink users are required to send acknowledgement packets during transmission to the FD BS, the FD BS can constantly update its CSI estimation of the links. Accordingly, the downlink users also receive the pilot signals of the uplink users and feedback the CSI between the uplink and downlink user to the FD BS.

Furthermore, due to the simultaneous transmission and reception at the FD $\mathrm{BS}$, there is a strong SI that degrades the reception of the uplink signals at the FD BS. In the literature, there are several SI mitigation techniques which could be employed to reduce the effects of SI. In order to isolate our proposed scheme from the specific implementation of any passive or active SI mitigation techniques, we model the SI after cancellation as $s_{j}=\rho\left\{\mathbf{u}_{j}^{H} \mathbf{H}_{\mathbf{S I}}\left(\sum_{k=1}^{K} \mathbf{W}_{k}\right) \mathbf{H}_{\text {SI }}^{H} \mathbf{u}_{j}\right\}$ [4], where the matrix $\mathbf{H}_{\mathbf{S I}} \in \mathbb{C}^{N \times N}$ denotes the SI channel at the FD BS and $0 \leq \rho \ll 1$ is the SI cancellation constant.

The FD BS has separate queue buffers to store data for each downlink user. Similarly, at each uplink user a queue buffer is used to store the data to be transmitted to the FD BS. Data arrive through a random process every slot at the FD BS and the uplink users. At slot $t$, the queue length for the $k$-th downlink user and the $j$-th uplink user are denoted by $Q_{\mathrm{DL}, k}(t)$ and $Q_{\mathrm{UL}, j}(t)$, respectively, and these queues evolve as follows:

$$
\begin{aligned}
& Q_{\mathrm{DL}, k}(t+1)=\max \left\{Q_{\mathrm{DL}, k}(t)-R_{\mathrm{DL}, k}(t), 0\right\}+A_{\mathrm{DL}, k}(t), \\
& Q_{\mathrm{UL}, j}(t+1)=\max \left\{Q_{\mathrm{UL}, j}(t)-R_{\mathrm{UL}, j}(t), 0\right\}+A_{\mathrm{UL}, j}(t),
\end{aligned}
$$

where $A_{\mathrm{DL}, k}(t)$ and $A_{\mathrm{UL}, j}(t)$ are the data arrival rate for the $k$-th downlink user and the $j$-th uplink user, which follow Poisson distributions, with mean arrival rates of $\bar{A}_{\mathrm{DL}, k}$ and $\bar{A}_{\mathrm{UL}, j}$, respectively. Accordingly, as data keeps arriving in every slot $t$, the queue buffers become overloaded which may result to packet drop. In essence, the queue buffers become unstable and communication becomes unreliable in the system.

As such, a discrete time queue process $Q(t)$ is mean-stable if $\lim _{t \rightarrow \infty} \frac{\mathbb{E}[|Q(t)|]}{t}=0$ [11], and a system of queues is said to be stable if all individual queues are stable. This implies that to stabilize the system, it is required to control the size of the queues. In addition, according to Little's law [6], for a given arrival rate the average queue length is proportional to the average delay. Thus, the system delay is dependent on queue length and stability. As a result, we can address the transmission delay by the queue length and stability. To this end, in our problem formulation we impose a queue stability constraint to ensure that the data in the buffers are not trapped and are delivered with a finite delay. This is equivalent to ensuring that the long-term average transmission rates are greater or equal to the average arrival rates.

\section{Delay-COnstrained Power Minimization AND ALGORITHM DESIGN}

Due to the stochastic nature of the channel conditions and data arrivals in the considered system and following [7]-[9], we consider the long-term average system performance metrics in our design. Thus, our main objective is to design an optimal resource allocation optimization problem that minimizes the the time-averaged total system transmit power while satisfying queue stability constraints, quality of service (QoS) constraints as well as maximum power constraints. This can be mathematically formulated as

$$
\begin{aligned}
& \text { P1 } \min _{\substack{\left\{\mathbf{W}_{k} \geq 0\right\} \\
\left\{P_{\mathrm{UL}, j}\right\}}} c_{1} \cdot \sum_{k=1}^{K} \bar{P}_{\mathrm{DL}, k}+c_{2} \cdot \sum_{j=1}^{J} \bar{P}_{\mathrm{UL}, j} \\
& \text { s.t. } \mathrm{A} 1: \bar{R}_{\mathrm{DL}, k} \geq \bar{A}_{\mathrm{DL}, k}, \forall k, \text { A } 2: \bar{R}_{\mathrm{UL}, j} \geq \bar{A}_{\mathrm{UL}, j}, \forall j, \\
& \text { A3 }: \gamma_{\mathrm{DL}, k}(t) \geq \Gamma_{\mathrm{DL}}, \forall k, \text { A4 }: \gamma_{\mathrm{UL}, j}(t) \geq \Gamma_{\mathrm{UL}}, \forall j, \\
& \text { A5 }: \sum_{k=1}^{K} P_{\mathrm{DL}, k}(t) \leq P_{\text {max }}^{\mathrm{DL}}, \mathrm{A} 6: 0 \leq P_{\mathrm{UL}, j}(t) \leq P_{\mathrm{max}}^{\mathrm{UL}}, \forall j,
\end{aligned}
$$


where $P_{\mathrm{DL}, k}(t)=\operatorname{Tr}\left\{\mathbf{W}_{k}\right\}$, and $c_{1}$ and $c_{2}$ are weights attached to the total downlink and uplink transmit powers, respectively. Here, we define $\bar{x}=\lim _{T \rightarrow \infty} \frac{1}{T} \sum_{t=1}^{T} \mathbb{E}[x(t)]$ as the time average expectation of the variable $x(t)$. Accordingly, constraints A1 and A2 ensure that the time-averaged transmission rate is greater or equal to the average arrival rate, which guarantee queue stability for the uplink and downlink queues. Constraints A3 and A4 ensure a minimum QoS for all users and constraint A5 and A6 impose the maximum power on the the FD BS and uplink users, respectively. The problem (7) is a non-convex problem due the time-averaged objective and constraints, thus, classified as a stochastic problem [11]. In order to solve (7) we resort to the classical drift-plus-penalty approach based on Lyapunov framework [11].

Accordingly, based on [11], we can transform the long-term problem to the following problem in each time slot. We refer the reader to [11] for the detailed derivation. Specifically, the optimisation problem is given by

$$
\begin{aligned}
\mathrm{P} 2: \max _{\substack{\left\{\mathbf{W}_{k} \geq 0\right\} \\
\left\{P_{\mathrm{UL}, j}\right\}}} \sum_{k=1}^{K} Q_{\mathrm{DL}, k}(t) R_{\mathrm{DL}, k}(t)+\sum_{j=1}^{J} Q_{\mathrm{UL}, j}(t) R_{\mathrm{UL}, j}(t) \\
-V \cdot\left(c_{1} \sum_{k=1}^{K} P_{\mathrm{DL}, k}(t)+c_{2} \sum_{j=1}^{J} P_{\mathrm{UL}, j}(t)\right)
\end{aligned}
$$

s.t. A3, A4, A5, A6.

The minimization problem (8) is not trivial to handle due to the rate expressions in the objective term. Thus, we simplify the objective as shown in (9) by expanding the non-linear log terms into linear log terms. The objective function in (9) is still non-convex, however, by close observation (9) can be written as a difference of two concave functions. Thus, in order to solve (8), we employ the convex-concave procedure (CCP) [12] to convexify the objective function. The basic idea of the CCP is to convexify the difference of convex (DC) problem by replacing the convex part of the function by their first order Taylor series expansions. This then makes the convex part affine functions. Hence, the CCP solves a series of concave problems successively and iteratively by initiating the procedure with a feasible point. In addition, the CCP has been proven to converge to the local optima of DC programming, we refer the reader to [12] for a formal proof.

Accordingly, the objective function in (9) can be written as a difference of two concave functions, i.e., $f_{\mathrm{DL}, k}(t)-r_{\mathrm{DL}, k}(t)$ and $f_{\mathrm{UL}, j}(t)-r_{\mathrm{UL}, j}(t)$, where,

$$
f_{\mathrm{DL}, k}(t)=\log _{2}\left(\sum_{i=1}^{K} \mathbf{h}_{k}^{H} \mathbf{W}_{i} \mathbf{h}_{k}+\sum_{j=1}^{J} P_{\mathrm{UL}, j}\left|\ell_{j, k}\right|^{2}+\sigma_{k}^{2}\right),
$$

$$
\begin{gathered}
r_{\mathrm{DL}, k}(t)=\log _{2}\left(\sum_{i \neq k}^{K} \mathbf{h}_{k}^{H} \mathbf{W}_{i} \mathbf{h}_{k}+\sum_{j=1}^{J} P_{\mathrm{UL}, j}\left|\ell_{j, k}\right|^{2}+\sigma_{k}^{2}\right), \\
f_{\mathrm{UL}, j}(t)=\log _{2}\left(s_{j}+\sigma_{j}^{2}\left\|\mathbf{u}_{j}\right\|^{2}+P_{\mathrm{UL}, j}\left|\mathbf{g}_{j}^{H} \mathbf{u}_{j}\right|^{2}\right), \\
r_{\mathrm{UL}, j}(t)=\log _{2}\left(s_{j}+\sigma_{j}^{2}\left\|\mathbf{u}_{j}\right\|^{2}\right) .
\end{gathered}
$$

Based on the above analysis, the optimization problem (8) can be reformulated as a standard DC problem as shown below

$$
\begin{aligned}
\widetilde{\mathrm{P} 2}: \max _{\substack{\left\{\mathbf{W}_{k} \geq 0\right\} \\
\left\{P_{\mathrm{UL}, j}\right\}}} & \sum_{k=1}^{K} Q_{\mathrm{DL}, k}(t)\left(f_{\mathrm{DL}, k}(t)-r_{\mathrm{DL}, k}(t)\right) \\
& +\sum_{j=1}^{J} Q_{\mathrm{UL}, j}(t)\left(f_{\mathrm{UL}, j}(t)-r_{\mathrm{UL}, j}(t)\right) \\
& -V \cdot\left(c_{1} \sum_{k=1}^{K} P_{\mathrm{DL}, k}(t)+c_{2} \sum_{j=1}^{J} P_{\mathrm{UL}, j}(t)\right)
\end{aligned}
$$$$
\text { s.t. A3, A4, A5, A6. }
$$

The difficulty in solving (10) lies in convexifying the concave components $r_{\mathrm{DL}, k}(t)$ and $r_{\mathrm{UL}, j}(t)$. To proceed, suppose that the values of $\mathbf{W}_{k}$ and $P_{\mathrm{UL}, j}$ at the $i$-th iteration are denoted by $\mathbf{W}_{k}^{(i)}$ and $P_{\mathrm{UL}, j}^{(i)}$. Since $r_{\mathrm{DL}, k}(t)$ and $r_{\mathrm{UL}, j}(t)$ are differentiable, thus, we can express their first order affine approximations as shown below, respectively.

$$
\begin{aligned}
& r_{\mathrm{DL}, k}^{(i)}(t)= r_{\mathrm{DL}, k}\left(\mathbf{W}_{k}^{(i)}, P_{\mathrm{UL}, j}^{(i)}\right) \\
&+\sum_{j=1}^{J}\left[\left(\Psi_{\mathrm{DL}, k}^{(i)}\right)^{-1}\left(P_{\mathrm{UL}, j}-P_{\mathrm{UL}, j}^{(i)}\right)\left|\ell_{j, k}\right|^{2}\right] \\
&+\sum_{i \neq k}^{K}\left[\left(\Psi_{\mathrm{DL}, k}^{(i)}\right)^{-1} \mathbf{h}_{k}^{H}\left(\mathbf{W}_{i}-\mathbf{W}_{k}^{(i)}\right) \mathbf{h}_{k}\right], \\
& r_{\mathrm{UL}, j}^{(i)}(t)= r_{\mathrm{UL}, j}\left(\mathbf{W}_{k}^{(i)}\right) \\
&+\sum_{k=1}^{K}\left[\frac{\rho \mathbf{u}_{j}^{H} \mathbf{H}_{\mathrm{SI}}\left(\mathbf{W}_{k}-\mathbf{W}_{k}^{(i)}\right) \mathbf{H}_{\mathrm{SI}}^{H} \mathbf{u}_{j}}{\left.\rho \mathbf{u}_{j}^{H} \mathbf{H}_{\mathrm{SI}}\left(\sum_{l=1}^{K} \mathbf{W}_{l}^{(i)}\right) \mathbf{H}_{\mathrm{SI}}^{H} \mathbf{u}_{j}+\sigma_{j}^{2}\left\|\mathbf{u}_{j}\right\|^{2}\right],}\right.
\end{aligned}
$$

where

$$
\Psi_{\mathrm{DL}, k}^{(i)}=\sum_{m \neq k}^{K} \mathbf{h}_{k}^{H} \mathbf{W}_{m}^{(i)} \mathbf{h}_{k}+\sum_{j=1}^{J} P_{\mathrm{UL}, j}^{(i)}\left|\ell_{j, k}\right|^{2}+\sigma_{k}^{2} .
$$

$$
\begin{aligned}
& \sum_{k=1}^{K} Q_{\mathrm{DL}, k}(t)\left[\log _{2}\left(\sum_{i=1}^{K} \mathbf{h}_{k}^{H} \mathbf{W}_{i} \mathbf{h}_{k}+\sum_{j=1}^{J} P_{\mathrm{UL}, j}\left|\ell_{j, k}\right|^{2}+\sigma_{k}^{2}\right)-\log _{2}\left(\sum_{i \neq k}^{K} \mathbf{h}_{k}^{H} \mathbf{W}_{i} \mathbf{h}_{k}+\sum_{j=1}^{J} P_{\mathrm{UL}, j}\left|\ell_{j, k}\right|^{2}+\sigma_{k}^{2}\right)\right] \\
& +\sum_{j=1}^{J} Q_{\mathrm{UL}, j}(t)\left[\log _{2}\left(s_{j}+\sigma_{j}^{2}\left\|\mathbf{u}_{j}\right\|^{2}+P_{\mathrm{UL}, j}\left|\mathbf{g}_{j}^{H} \mathbf{u}_{j}\right|^{2}\right)-\log _{2}\left(s_{j}+\sigma_{j}^{2}\left\|\mathbf{u}_{j}\right\|^{2}\right)\right]-V \cdot\left(c_{1} \sum_{k=1}^{K} P_{\mathrm{DL}, k}(t)+c_{2} \sum_{j=1}^{J} P_{\mathrm{UL}, j}(t)\right)
\end{aligned}
$$




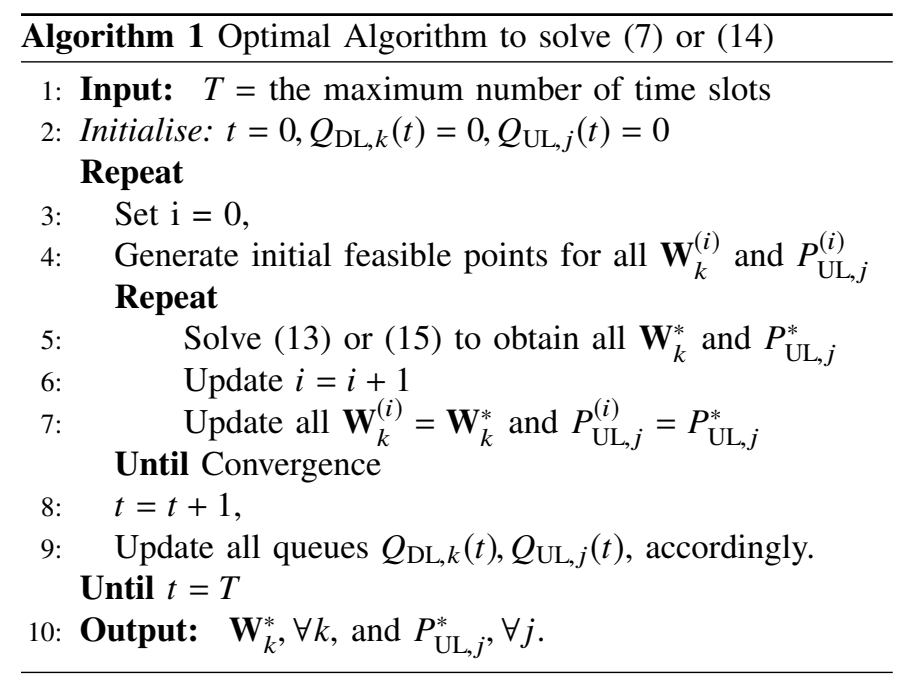

Here, we have used the fact that $\nabla_{\mathbf{X}} \log _{2}\left(\mathbf{I}+\mathbf{Z X} \mathbf{Z}^{H}\right)=$ $\mathbf{X}^{H}\left(\mathbf{I}+\mathbf{Z X Z} \mathbf{Z}^{H}\right)^{-1} \mathbf{X}$ and $\nabla_{x} \log _{2}(1+z x)=x(1+z x)^{-1}$. Therefore, the transformed optimization problem (10) can be expressed as

$$
\begin{aligned}
\mathrm{P} 3: \max _{\substack{\left\{\mathbf{W}_{k} \geq 0\right\} \\
\left\{\boldsymbol{P}_{\mathrm{UL}, j}\right\}}} & \sum_{k=1}^{K} Q_{\mathrm{DL}, k}(t)\left(f_{\mathrm{DL}, k}(t)-r_{\mathrm{DL}, k}^{(i)}(t)\right) \\
& +\sum_{j=1}^{J} Q_{\mathrm{UL}, j}(t)\left(f_{\mathrm{UL}, j}(t)-r_{\mathrm{UL}, j}^{(i)}(t)\right) \\
& -V \cdot\left(c_{1} \sum_{k=1}^{K} P_{\mathrm{DL}, k}(t)+c_{2} \sum_{j=1}^{J} P_{\mathrm{UL}, j}(t)\right)
\end{aligned}
$$

s.t. A3, A4, A5, A6.

Problem (13) is convex with respect to the optimization variables and can be solved efficiently using standard convex solvers. Please note that the formulation in (13) is a relaxed problem where the rank 1 constraint on $\mathbf{W}_{k}$ has been dropped. If the resulting solution $\mathbf{W}_{k}$ after solving (13) is rank 1, the optimal $\mathbf{w}_{k}$ can be obtained by applying eigenvaluedecomposition (EVD), otherwise, randomization technique can be used to retrieve $\mathbf{w}_{k}$.

After obtaining the beamforming vectors and the transmit powers from (13) for slot $t$, the queues $Q_{\mathrm{DL}, k}(t), Q_{\mathrm{UL}, j}(t)$ are updated accordingly. The overall procedure to solve the optimization problem (7) is summarized in Algorithm 1.

\section{DELAY FAIRnESS Optimization}

Building upon the simplified problem formulation P3 in Section III, in this section, we propose a resource allocation algorithm based on delay fairness. By close examination of the simplified convex optimization problem P3, it can be observed that P3 basically aims at maximizing the sum rate of the uplink and downlink users while minimizing the sum transmit power. Resource allocation that focuses solely on sum rate maximization usually results in an unfair resource allocation, since the system resources are consumed by the users with good channel conditions. In essence, the resource allocation

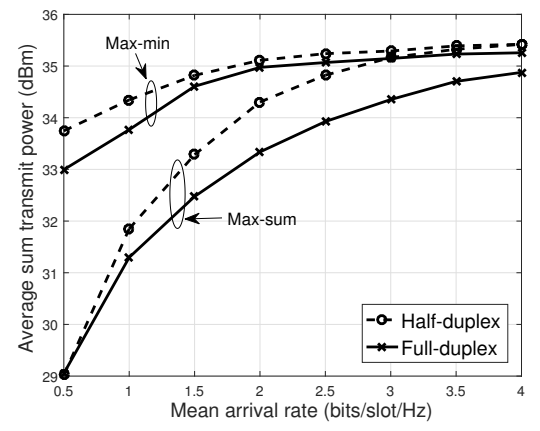

Fig. 2: Average sum transmit power versus mean arrival rate with $\Gamma_{\mathrm{DL}}=8 \mathrm{~dB}, \Gamma_{\mathrm{UL}}=6 \mathrm{~dB}$ and $V=4$

based on P3 results in starvation of users with poor channel conditions, which is unfair. As such, we formulate a fair beamforming optimization and resource allocation problem that aims at maximizing the minimum transmission rate for the downlink and uplink users, respectively. This problem is often referred to as the max-min problem and can be mathematically expressed as

$$
\begin{aligned}
& \mathrm{P} 4: \max _{\substack{\left\{\mathbf{W}_{k} \geq 0\right\} \\
\left\{P_{\mathrm{UL}, j}\right\}}} \min _{k}\left(Q_{\mathrm{DL}, k}(t)\left(f_{\mathrm{DL}, k}(t)-r_{\mathrm{DL}, k}^{(i)}(t)\right)\right) \\
&+\min _{j}\left(Q_{\mathrm{UL}, j}(t)\left(f_{\mathrm{UL}, j}(t)-r_{\mathrm{UL}, j}^{(i)}(t)\right)\right) \\
&-V \cdot\left(c_{1} \sum_{k=1}^{K} P_{\mathrm{DL}, k}(t)+c_{2} \sum_{j=1}^{J} P_{\mathrm{UL}, j}(t)\right)
\end{aligned}
$$$$
\text { s.t. A3, A4, A5, A6. }
$$

The max-min problem P4 can not be solved directly in it's current form, however, P4 can be simplified by introducing auxilary variables [13], $t_{\mathrm{DL}}(t)$ and $t_{\mathrm{UL}}(t)$, respectively. Thus, the simplified convex problem is given by

$$
\begin{gathered}
\widetilde{\mathrm{P} 4}: \max _{\substack{\left\{\mathbf{W}_{k} \geq 0\right\} \\
\left\{P_{\mathrm{UL}, j}\right\}}} t_{D L}(t)+t_{U L}(t)-V \cdot\left(c_{1} \sum_{k=1}^{K} P_{\mathrm{DL}, k}(t)+c_{2} \sum_{j=1}^{J} P_{\mathrm{UL}, j}(t)\right) \\
\text { s.t. } \quad Q_{\mathrm{DL}, k}(t)\left(f_{\mathrm{DL}, k}(t)-r_{\mathrm{DL}, k}^{(i)}(t)\right) \geq t_{\mathrm{DL}}(t), \forall k, \\
Q_{\mathrm{UL}, j}(t)\left(f_{\mathrm{UL}, j}(t)-r_{\mathrm{UL}, j}^{(i)}(t)\right) \geq t_{\mathrm{UL}}(t), \forall j,
\end{gathered}
$$$$
\text { A3, A4, A5, A6. }
$$

The problem (15) is convex and can be efficiently solved using standard solvers. Similar to (13), the formulation (15) is a relaxed problem. If the resulting solution $\mathbf{W}_{k}$ after solving (13) is rank 1 , the optimal $\mathbf{w}_{k}$ can be obtained by applying eigenvalue-decomposition (EVD), otherwise, randomization technique can be used to retrieve $\mathbf{w}_{k}$. The procedure for solving (14) is summarized in Algorithm 1.

\section{Simulation Results}

We consider the system with the FD BS at the centre of a cell with $N=3$ antennas, each for transmitting and 


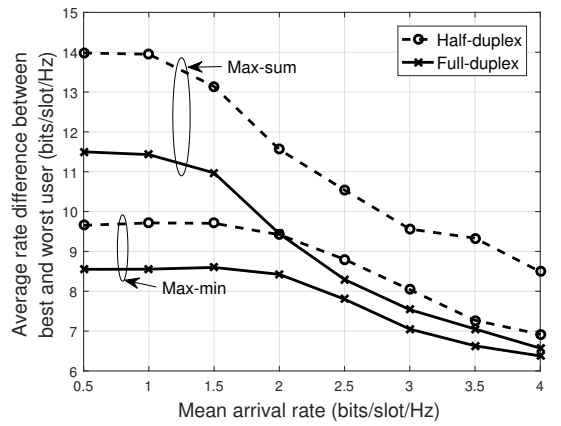

Fig. 3: Average rate difference between the best and worst user with $\Gamma_{\mathrm{DL}}=8 \mathrm{~dB}, \Gamma_{\mathrm{UL}}=6 \mathrm{~dB}$ and $V=4$

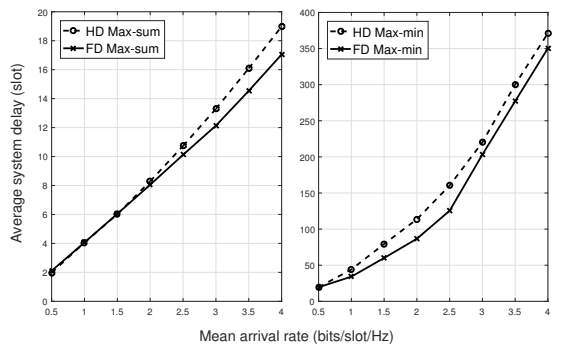

Fig. 4: Average system delay versus mean arrival rate with $\Gamma_{\mathrm{DL}}=8 \mathrm{~dB}, \Gamma_{\mathrm{UL}}=6 \mathrm{~dB}$ and $V=4$

receiving. We assume $K=J=2$ downlink and uplink users, are randomly and uniformly distributed between the distance of $10 \mathrm{~m}$ and $50 \mathrm{~m}$. We model the channels to the downlink and uplink users as Rayleigh fading. The SI channel is modelled as Rician fading channel with Rician factor $6 \mathrm{~dB}$. Furthermore, we consider a similar system set-up as in [4] with $\sigma_{i}=\sigma_{j}=-60 \mathrm{dBm}, P_{\max }^{\mathrm{UL}}=23 \mathrm{dBm}, P_{\max }^{\mathrm{DL}}=35 \mathrm{dBm}, \rho=$ $-80 \mathrm{~dB}, c_{1}=c_{2}=1$ and we assume $T=10000$ slots to approximate $t \rightarrow \infty$. For comparison we adopt the HD technique as in [7]-[9]. For fair comparison, here, the overall data rate of HD is set equal to the one for FD which requires that the individual data rate of the downlink and uplink users are double the ones for the FD case, due to the slotted HD transmission. Besides, the CCI and SI are avoided with HD. In the figures, we refer to the results obtained from the problem formulations in (7) and (14) as max-sum and maxmin, respectively.

In Fig. 2 we show the average sum transmit power for varying mean arrival rate. It can be seen that the average sum transmit power increases as the mean arrival rate increases for both the proposed FD max-sum and max-min schemes, since an increase in arrival rate implies more data to be transmitted which requires an increase in the transmit power. In addition, the proposed max-sum optimization achieves the minimum sum transmit power compared with the proposed max-min optimization. This is because the proposed max-min optimization tries to balance the power allocation among all users, since some users perform poorer than others. This is further illustrated in Fig. 3, where the average rate difference between the best and worst user is plotted against the mean arrival rate. It can be seen that the proposed max-min optimization has a lower rate difference since it gives no preference in the power allocation between all users. Moreover, the figure also shows that the proposed FD schemes out perform the baseline HD schemes for both max-sum and max-min resource allocation.

Furthermore, Fig. 4 shows the average system delay in slots with varying mean arrival rate for the proposed FD max-sum and max-min resource allocation schemes, respectively. Here we define the delay as the queue length for each user in each time slot and thus, the average system delay is the total system delay for all users averaged over the considered maximum number of time slots $T$. As can be observed, the system delay is proportional to increase in arrival rates. The proposed maxsum scheme achieves lower delay because it exploits users with good channel conditions to improve the system delay, while the proposed max-min ensures fairness to all users at the expense of higher system delay.

\section{CONCLUSION}

In this paper, we studied the delay-constrained beamforming and resource allocation problem in a multiuser FD system. We minimized the long-term sum transmit power while satisfying the long-term stability constraints on the queue buffers.

\section{REFERENCES}

[1] M. Duarte, A. Sabharwal, V. Aggarwal, R. Jana, K. Ramakrishnan, C. W. Rice, and N. Shankaranarayanan, "Design and characterization of a fullduplex multiantenna system for WiFi networks," IEEE Transactions on Vehicular Technology, vol. 63, no. 3, pp. 1160-1177, 2014.

[2] M. Bengtsson and B. Ottersten, "Handbook of antennas in wireless communications," Optimal and Suboptimal Transmit Beamforming: Boco Raton, FL: CRC, 2001.

[3] D. W. K. Ng, Y. Wu, and R. Schober, "Power efficient resource allocation for full-duplex radio distributed antenna networks," IEEE Transactions on Wireless Communications, vol. 15, no. 4, pp. 2896-2911, 2016.

[4] Y. Sun, D. W. K. Ng, J. Zhu, and R. Schober, "Multi-objective optimization for robust power efficient and secure full-duplex wireless communication systems," IEEE Transactions on Wireless Communications, vol. 15 , no. 8, pp. 5511-5526, 2016

[5] M. T. Kabir, M. R. Khandaker, and C. Masouros, "Interference exploitation in full-duplex communications: Trading interference power for both uplink and downlink power savings," IEEE Transactions on Wireless Communications, vol. 17, no. 12, pp. 8314-8329, 2018.

[6] J. D. Little, "A proof for the queuing formula: $\mathrm{L}=\lambda \mathrm{w}$," Operations research, vol. 9, no. 3, pp. 383-387, 1961.

[7] Y. Li, M. Sheng, Y. Shi, X. Ma, and W. Jiao, "Energy efficiency and delay tradeoff for time-varying and interference-free wireless networks," IEEE Transactions on Wireless Communications, vol. 13, no. 11, pp. 5921-5931, 2014.

[8] J. Hajipour, J. M. Niya, and D. W. K. Ng, "Energy-efficient resource allocation in buffer-aided wireless relay networks," IEEE Transactions on Wireless Communications, vol. 16, no. 10, pp. 6648-6659, 2017.

[9] H. Ju, B. Liang, J. Li, and X. Yang, "Dynamic joint resource optimization for lte-advanced relay networks," IEEE Transactions on Wireless Communications, vol. 12, no. 11, pp. 5668-5678, 2013.

[10] B. Li, H. H. Dam, A. Cantoni, and K. L. Teo, "Some interesting properties for zero-forcing beamforming under per-antenna power constraints in rural areas," Journal of Global Optimization, vol. 62, no. 4, pp. 877886, 2015.

[11] M. J. Neely, "Stochastic network optimization with application to communication and queueing systems," Synthesis Lectures on Communication Networks, vol. 3, no. 1, pp. 1-211, 2010.

[12] T. Lipp and S. Boyd, "Variations and extension of the convex-concave procedure," Optimization and Engineering, vol. 17, no. 2, pp. 263-287, 2016.

[13] S. Boyd and L. Vandenberghe, Convex optimization. Cambridge university press, 2004. 